九州大学学術情報リポジトリ

Kyushu University Institutional Repository

\title{
Patterns of practice in intensity-modulated radiation therapy and image-guided radiation therapy for prostate cancer in Japan
}

Nakamura, Katsumasa

Department of Clinical Radiology, Graduate School of Medical Sciences, Kyushu University

Akimoto, Tetsuo

Department of Radiation Oncology and Particle Therapy, National Cancer Center Hospital East

Mizowaki, Takashi

Department of Radiation Oncology and Image-applied Therapy, Kyoto University Graduate School of Medicine

Hatano, Kazuo

Division of Radiation Oncology, Chiba Cancer Center

他

http://hdl. handle. net/2324/27281

出版情報: Japanese Journal of Clinical Oncology. 42 (1), pp.53-57，2011-11-30. Oxford University Press

バージョン:

権利関係: (C) The Author 2011. Published by 0xford University Press 
Patterns of practice in intensity-modulated radiation therapy and image-guided radiation therapy for prostate cancer in Japan.

Katsumasa Nakamura ${ }^{1}$, Tetsuo Akimoto ${ }^{2}$, Takashi Mizowaki ${ }^{3}$, Kazuo Hatano ${ }^{4}$, Takeshi Kodaira ${ }^{5}$, Naoki Nakamura ${ }^{6}$, Takuyo Kozuka ${ }^{7}$, Naoto Shikama ${ }^{8}$, and Yoshikazu Kagami $^{9}$

1. Department of Clinical Radiology, Graduate School of Medical Sciences, Kyushu University, Fukuoka, Japan

2. Department of Radiation Oncology and Particle Therapy, National Cancer Center Hospital East, Kashiwa, Japan

3. Department of Radiation Oncology and Image-applied Therapy, Kyoto University Graduate School of Medicine, Kyoto, Japan

4. Division of Radiation Oncology, Chiba Cancer Center, Chiba, Japan

5. Department of Radiation Oncology, Aichi Cancer Center, Nagoya, Japan.

6. Department of Radiation Oncology, St Luke's International Hospital, Tokyo, Japan

7. Department of Radiation Oncology, The Cancer Institute, Ariake Hospital of Japanese Foundation for Cancer Research, Tokyo, Japan 
8. Department of Radiation Oncology, Saitama Medical University International Medical Center, Saitama, Japan

9. Department of Radiology, Showa University School of Medicine, Tokyo, Japan

Correspondence to: Katsumasa Nakamura, Department of Clinical Radiology, Graduate

School of Medical Sciences, Kyushu University, Maidashi 3-1-1, Higashi-ku, Fukuoka 812-8582, Japan.

Tel. 092-642-5695; Fax 092-642-5708

E-mail: nakam@radiol.med.kyushu-u.ac.jp

Running head: Patterns of radiotherapy for Prostate Cancer 
Abstract

Background: The purpose of this study was to compare the prevalence of treatment techniques including intensity-modulated radiation therapy (IMRT) and image-guided radiation therapy (IGRT) in external-beam radiation therapy (EBRT) for prostate cancer in Japan.

Methods: A national survey on the current status of EBRT for prostate cancer was performed in 2010. We sent questionnaires to 139 major radiotherapy facilities in Japan, of which 115 (82.7\%) were returned.

Results: IMRT was conducted at 67 facilities (58.3\%) while IGRT was conducted at 70 facilities (60.9\%). Simulations and treatments were performed in the supine position at most facilities. In two-thirds of the facilities, a filling bladder was requested. Approximately $80 \%$ of facilities inserted a tube or encouraged defecation when the rectum was dilated. Some kind of fixation method was used at 102 facilities (88.7\%). Magnetic resonance imaging (MRI) was routinely performed for treatment planning at 32 facilities (27.8\%). The median total dose was 76 Gy with IMRT and 70 Gy with 3DCRT. The doses were prescribed at the isocenter at the facilities that conducted 3DCRT. In contrast, the dose prescription varied at the facilities that conducted IMRT. 
Of the 70 facilities that could perform IGRT, 33 (47.1\%) conducted bone matching, 28 (40.0\%) conducted prostate matching, and 9 (12.9\%) used metal markers. Prostate or metal marker matching tended to produce a smaller margin than bone matching.

Conclusion: The results of the survey identified current patterns in the treatment planning and delivery processes of EBRT for prostate cancer in Japan.

Mini-abstract: A national survey on the current status of radiotherapy for prostate cancer identified the patterns in the treatment planning and delivery processes of radiotherapy for prostate cancer in Japan. 
Introduction

External beam radiation therapy (EBRT) has developed rapidly in recent years $(1,2)$, and treatment equipment with which intensity-modulated radiation therapy (IMRT) and/or image-guided radiation therapy (IGRT) can be conducted are being introduced into Japan (3). IMRT and IGRT are particularly useful in EBRT for prostate cancer, and are routinely used in the United States (4), and recommended in worldwide guidelines $(5,6)$.

In Japan, IMRT and IGRT were listed as eligible for insurance reimbursement in 2008 and 2010, respectively. However, the present situation regarding the use of these techniques in EBRT for prostate cancer remains unclear $(7,8)$. Therefore, we conducted a survey that would clarify the operational situation, treatment planning and treatment processes of IMRT and/or IGRT when used in EBRT for prostate cancer.

Materials and Methods

In February 2010, we sent a questionnaire on EBRT for prostate cancer to 139 major facilities including university hospitals, cancer centers and designated prefectural 
cancer centers and hospitals. The questionnaire was also sent to the hospitals which had treatment machines with IGRT functions including Novalis (BrainLAB, Heimstetten, Germany), Tomotherapy (Accuray Inc., Sunnyvale, USA) and MHI-TM2000 (Mitsubishi Heavy Industries, Ltd., Nagoya, Japan).

The survey was composed of categories regarding treatment planning, dose fractionation and methods of implementation of EBRT for prostate cancer. If methods differed according to the type of radiation techniques used such as three-dimensional radiation therapy (3DCRT) or IMRT, we required responses regarding the most precise radiation method presently used. Among the 139 facilities to which we sent the survey, 115 (82.7\%) gave responses, which were then analyzed. The high response rate allowed an extensive and representative data analysis.

Results

General information

Figure 1 shows the distribution of the number of patients with prostate cancer treated with EBRT at facilities in 2009 over the course of one year. There were 30 facilities (26.1\%) at which over 50 patients were treated in one year. Of the 115 total 
facilities, 67 (58.3\%) conducted IMRT, 70 (60.9\%) conducted IGRT, and 58 (50.4\%) conducted both.

Treatment planning

Figure 2 shows the condition of the bladder at the treatment planning and the treatment. In approximately two-thirds of the facilities, a filling bladder was requested. The time spent pooling urine was 1 hour at 56 facilities (48.7\%), 1 to 2 hours at 8 facilities (7.0\%), and 30 minutes at 7 facilities (6.1\%). Seven facilities (6.1\%) also asked patients to drink water prior to treatment.

Figure 3 shows the condition of the rectum. Approximately $80 \%$ of facilities inserted a tube or encouraged defecation when the rectum was dilated. Laxative medication was used at one-quarter of the facilities.

Simulations and treatments were performed in the supine position at 105 facilities (91.3\%) and the prone position at 10 facilities (8.7\%). Figure 4 shows methods of patient fixation. Some kind of fixation method was used at 102 facilities (88.7\%). Although various methods were reported, a vacuum cushion, thermoplastic shell and foot support were used most frequently.

Magnetic resonance imaging (MRI) was routinely performed for treatment 
planning at 32 facilities (27.8\%). Of these, 15 facilities (13.0\%) performed CT-MRI image fusion with treatment planning software. MRI taken at the time of diagnosis was used as a reference at 66 facilities (57.4\%) while 17 facilities (14.8\%) did not use MRI for treatment planning.

Treatment

Radiation therapy was carried out with 2 Gy per fraction at 100 facilities (86.9\%), 2.1 to 3 Gy at 14 facilities (12.2\%) and 1.8 Gy at 1 facility (0.9\%). Most facilities conducted treatment 5 times a week. Treatment was conducted 3 times a week at 5 facilities (4.3\%) and 4 times a week at 3 facilities (2.6\%).

Figure 5 shows the distributions of radiation doses delivered to the prostate at facilities using a fraction dose of 2 Gy. The median total dose was 76 Gy with IMRT and 70 Gy with 3DCRT. The doses were prescribed at the isocenter at the facilities that conducted 3DCRT. In contrast, the dose prescription varied greatly at the facilities that conducted IMRT. Of the 67 facilities that conducted IMRT, D95, which is the minimum absorbed dose that covers 95\% of the planning target volume (PTV), was used as a dose prescription at 24 facilities (35.8\%). A dose prescription requiring that $95 \%$ of the prescribed isodose line cover 95\% of the PTV was used at 4 facilities (6.0\%), the mean 
PTV dose was used at 13 facilities (19.4\%), and other methods at 26 facilities (38.8\%).

The most popular IGRT methods (54 facilities) involved 2D matching with X-ray fluoroscopy or 3D matching with flat-panel cone-beam CT. Eight facilities used CT on rail, and 4 facilities used ultrasonic devices. Of the 70 facilities that could perform IGRT, 33 (47.1\%) conducted bone matching, 28 (40.0\%) conducted prostate matching, and 9 (12.9\%) used metal markers. At the treatment of prostate cancer, 60 facilities (85.7\%) always conducted IGRT, while 9 (12.9\%) conducted IGRT at regular intervals.

Figures 6 show the distribution of the prostate-PTV margins for patients with typical T1-2 tumors treated with IGRT. Prostate or metal marker matching tended to produce slightly smaller margins than bone matching.

Discussion

This study provides a clear picture of present practices of IMRT and/or IGRT for prostate cancer in Japan.

Simulations and treatments were performed in the supine position at most facilities. However, facilities employed various fixation methods. In most facilities some kind of fixation method was used, although immobilization devices for body 
malignancies are not covered by health insurance in Japan. In the Patterns of Care Study on prostate cancer patients who were treated with EBRT from 2003 to 2005, immobilization devices were used on only $15 \%$ of patients (7). One reason for the high frequency of the usage of patient immobilization devices in this study could be the gradual popularization of fixation methods over time. An additional reason is probably the fact that some sort of fixation method tends to be used in more precise radiation treatment, because patient immobilization can be an important contributor to the reproducibility and accuracy of radiotherapy (9).

The pretreatment condition of the bladder and rectum also varied greatly among facilities. Although fixation of the prostate is frequently conducted with a rectal balloon in Western countries (10), this method has not been used at all in Japan. In this study, we did not investigate PTV margins when IGRT was not used. Therefore, we were unable to clarify whether IGRT causes decreased margins. However, PTV margins tended to be slightly smaller with prostate or fiducial marker matching than with bone matching. PTV margins should be determined at each facility taking into account position errors caused not only by the IGRT method, but also by the patient position, fixation method and pretreatment condition of the bladder and rectum. Enmark et al. demonstrated that a margin of $4 \mathrm{~mm}$ in all directions was adequate to account for 
uncertainties including the inter- and intrafraction motions, if IGRT with fiducial markers is performed on a daily basis (11). Some facilities have chosen prostate-PTV margins of less than $4 \mathrm{~mm}$. Because of uncertainties such as intrafraction motion or uncertainty of the target delineation, decreases in the PTV margin should be carefully performed even when IGRT is applied.

The radiation dose administered at most facilities was 2 Gy per fraction. The median value of the total radiation dose was 76 Gy with IMRT and 70 Gy with 3DCRT. It is well known that that radiation dose is a strong independent predictor of failure (12), and IMRT can reduce the unwanted doses to nearby organs at risk. Therefore, as IMRT becomes more widespread in Japan, more appropriate higher dosages of radiation should be utilized. However, a significant problem is the fact that the IMRT dose prescription varies. It is necessary to define and develop recommended guidelines for dose prescription and a dose reporting system for IMRT in Japan (13).

IMRT and IGRT were being conducted at approximately half of the facilities in this study. However, our survey targeted large-scale facilities. If all radiation therapy facilities in Japan were to be surveyed, this proportion would probably be smaller (3). At present, high-precision radiation therapy devices such as IMRT and IGRT are being rapidly introduced $(3,14)$, and an increasing number of facilities will surely come to 
adopt IMRT and IGRT. The results of the survey in this study will provide beneficial information to those facilities as they begin treatment.

Acknowledgments

We thank all radiation oncologists who participated in this study. We are also grateful to Y. Asazawa for making the questionnaire available to us.

Funding

This study was supported in part by Grant-in Aid from the Ministry of Health, Labor and Welfare of Japan [H21-018]. 
References

1. Harmenberg U, Hamdy FC, Widmark A, Lennernas B, Nilsson S. Curative radiation therapy in prostate cancer. Acta Oncol. 2011;50 Suppl 1:98-103.

2. Ghilezan M, Yan D, Martinez A. Adaptive radiation therapy for prostate cancer. Semin Radiat Oncol. 2010;20:130-7.

3. Teshima T, Numasaki H, Shibuya H, Nishio M, Ikeda H, Sekiguchi K et al. Japanese structure survey of radiation oncology in 2007 based on institutional stratification of patterns of care study. International journal of radiation oncology, biology, physics. 2010;78:1483-93.

4. Kupelian P, Meyer JL. Image-guided, adaptive radiotherapy of prostate cancer: toward new standards of radiotherapy practice. Front Radiat Ther Oncol. 2011;43:344-68.

5. National Comprehensive Cancer Network. NCCN Clinical Practice Guidelines in Oncology: Prostate Cancer V1, 2011. Available at: http://www.nccn.org/

6. Horwich A, Parker C, Kataja V. Prostate cancer: ESMO clinical recommendations for diagnosis, treatment and follow-up. Ann Oncol. 2009;20 Suppl 4:76-8.

7. Nakamura K, Ogawa K, Sasaki T, Onishi H, Koizumi M, Araya M et al. Patterns of 
radiation treatment planning for localized prostate cancer in Japan: 2003-05 patterns of care study report. Jpn J Clin Oncol. 2009;39:820-4.

8. Ogawa K, Nakamura K, Sasaki T, Onishi H, Koizumi M, Araya M et al. Radical External Beam Radiotherapy for Clinically Localized Prostate Cancer in Japan: Changing Trends in the Patterns of Care Process Survey. International journal of radiation oncology, biology, physics. 2011; in press.

9. Fiorino C, Reni M, Bolognesi A, Bonini A, Cattaneo GM, Calandrino R. Set-up error in supine-positioned patients immobilized with two different modalities during conformal radiotherapy of prostate cancer. Radiother Oncol. 1998;49:133-41.

10. Smeenk RJ, Teh BS, Butler EB, van Lin EN, Kaanders JH. Is there a role for endorectal balloons in prostate radiotherapy? A systematic review. Radiother Oncol. 2010;95:277-82.

11. Enmark M, Korreman S, Nystrom H. IGRT of prostate cancer; is the margin reduction gained from daily IG time-dependent? Acta Oncol. 2006;45:907-14.

12. Viani GA, Stefano EJ, Afonso SL. Higher-than-conventional radiation doses in localized prostate cancer treatment: a meta-analysis of randomized, controlled trials. International journal of radiation oncology, biology, physics. 2009;74:1405-18.

13. ICRU. International Commission on Radiation Units and Measurements. 
Prescribing, Recording, and Reporting Photon-Beam Intensity-Modulated Radiation Therapy (IMRT). Report 83. Oxford: Oxford University Press; 2010.

14. Teshima T, Numasaki H, Shibuya H, Nishio M, Ikeda H, Ito $H$ et al. Japanese structure survey of radiation oncology in 2005 based on institutional stratification of patterns of care study. International journal of radiation oncology, biology, physics. 2008;72:144-52. 
Legends of figures

Figure 1. Total number of patients with prostate cancer treated with external-beam radiation therapy at facilities in 2009.

Figure 2. Condition of the bladder at treatment planning and treatment.

Figure 3. Condition of the rectum at treatment planning and treatment.

Figure 4. Fixation of the patients at planning and treatment.

Figure 5. Total dose to the prostate.

Figure 6. Margins from the prostate to PTV for patients with T1-2 tumors treated with IGRT; (a) rectal side and (b) other sides. 


\section{Figure 1}

No. of Hospitals

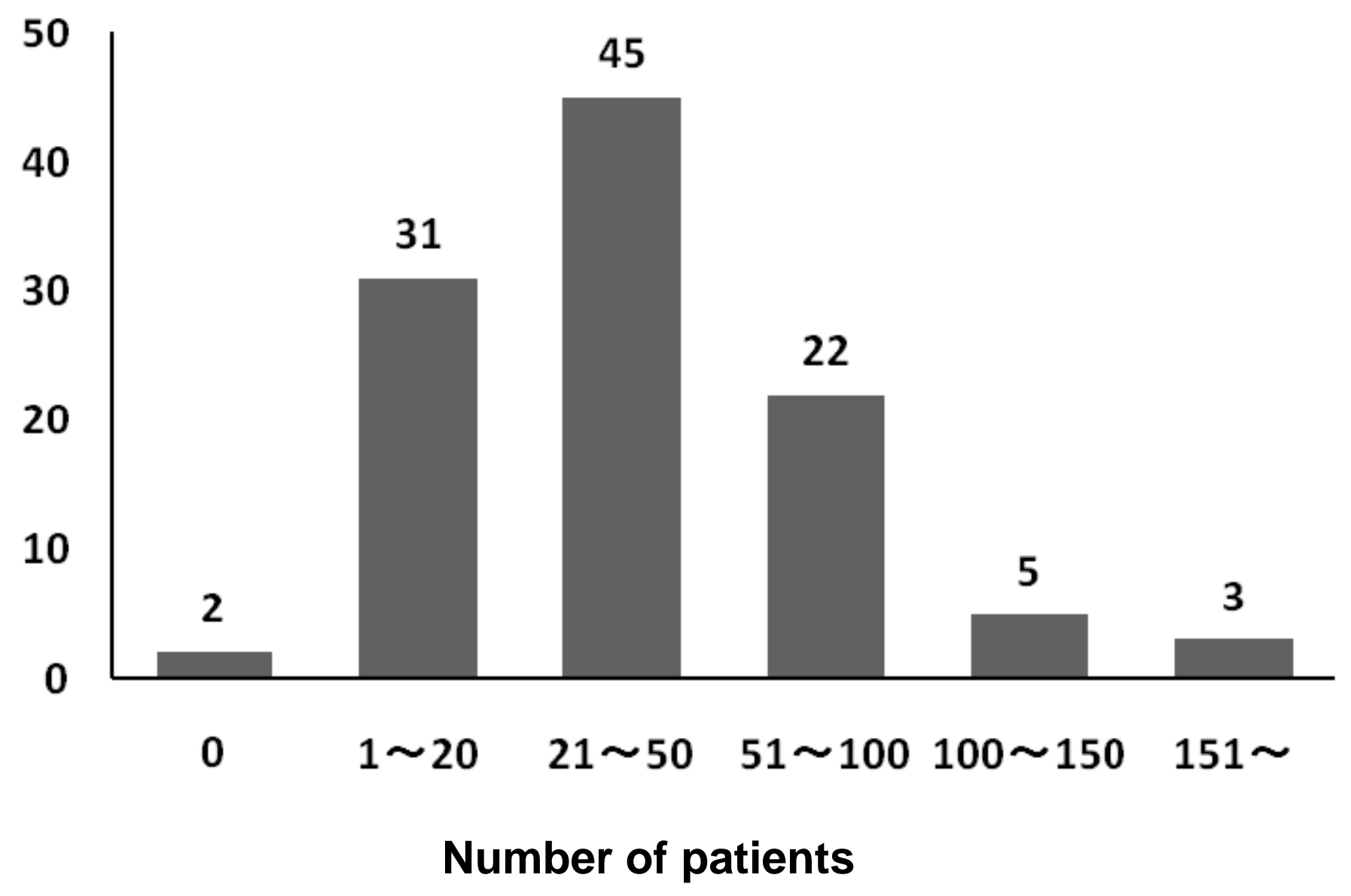

* Because some data were missing, the total numbers of patients were less than actual number. 


\section{Figure 2}

No. of Hospitals

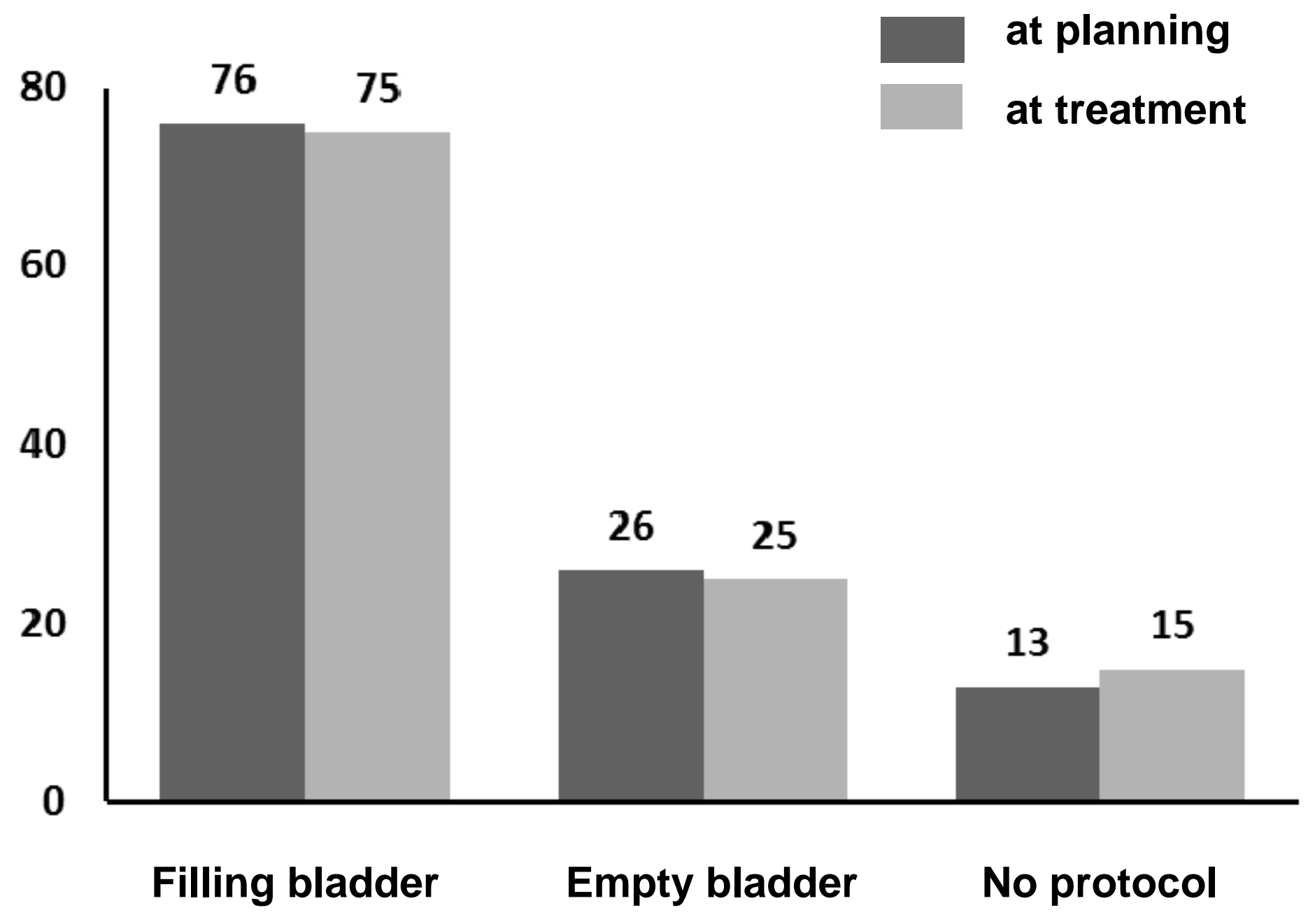


Figure 3

No. of Hospitals

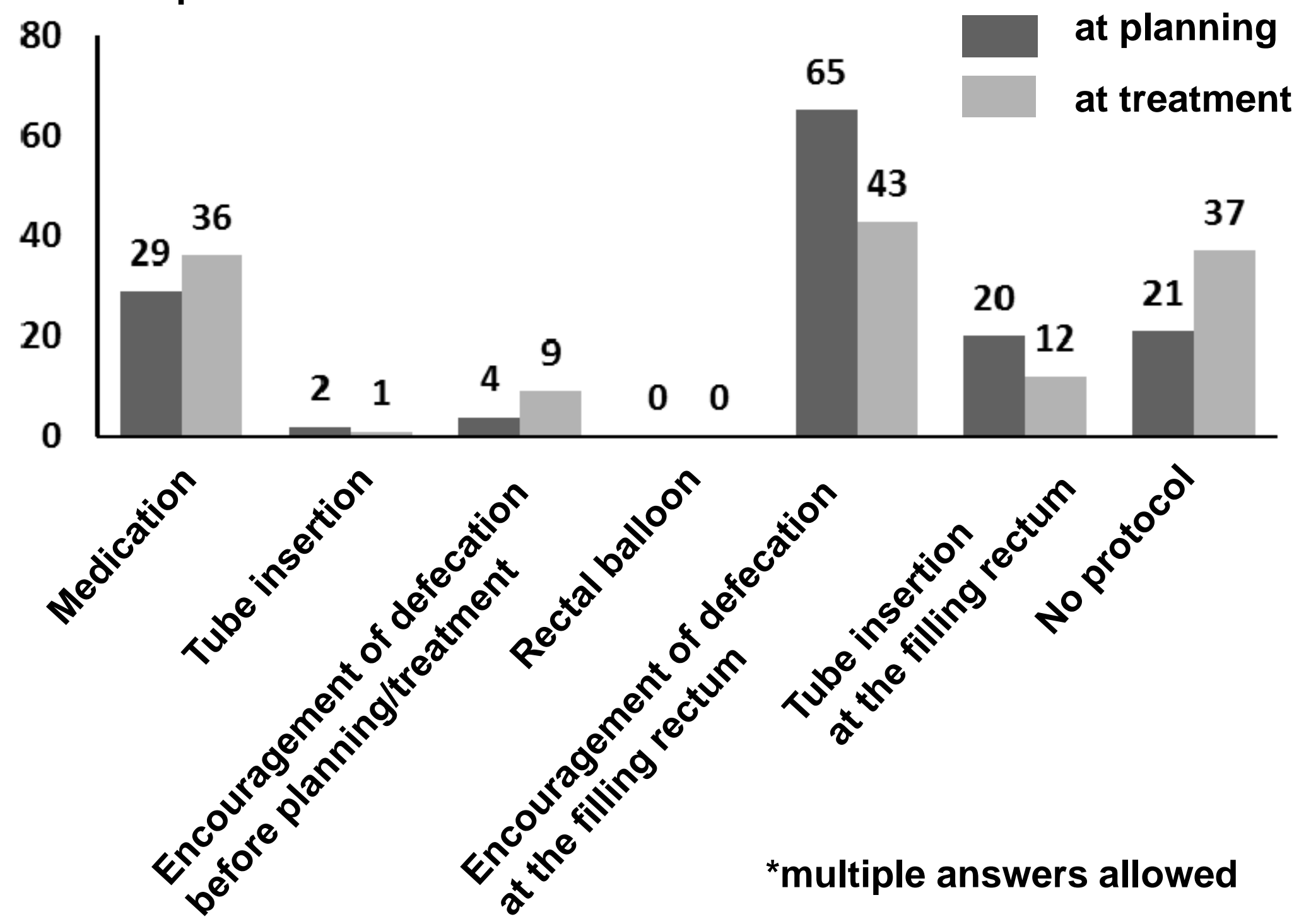


Figure 4

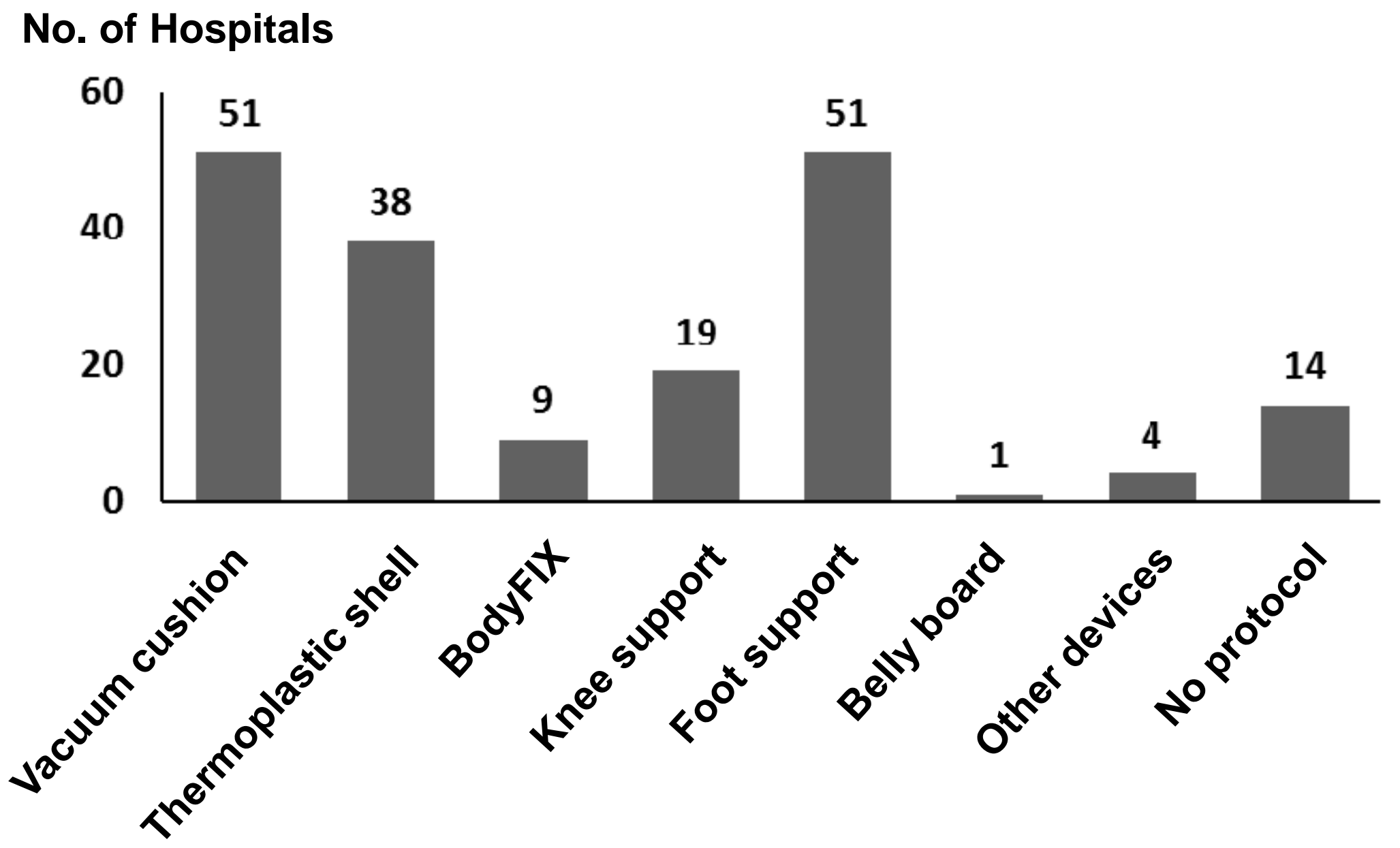

*multiple answers allowed 
Figure 5

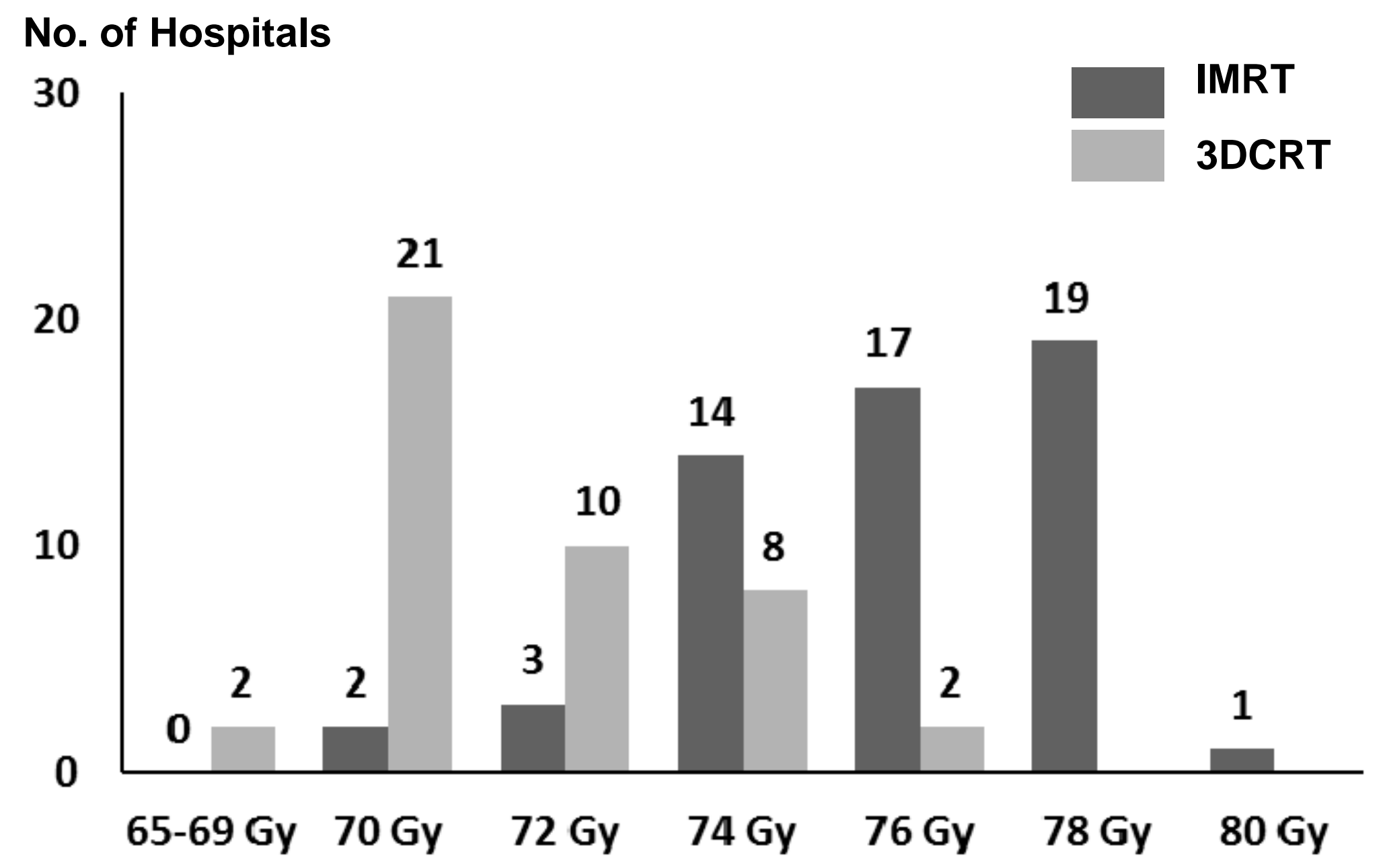




\section{Figure 6}

(a)

No. of Hospitals

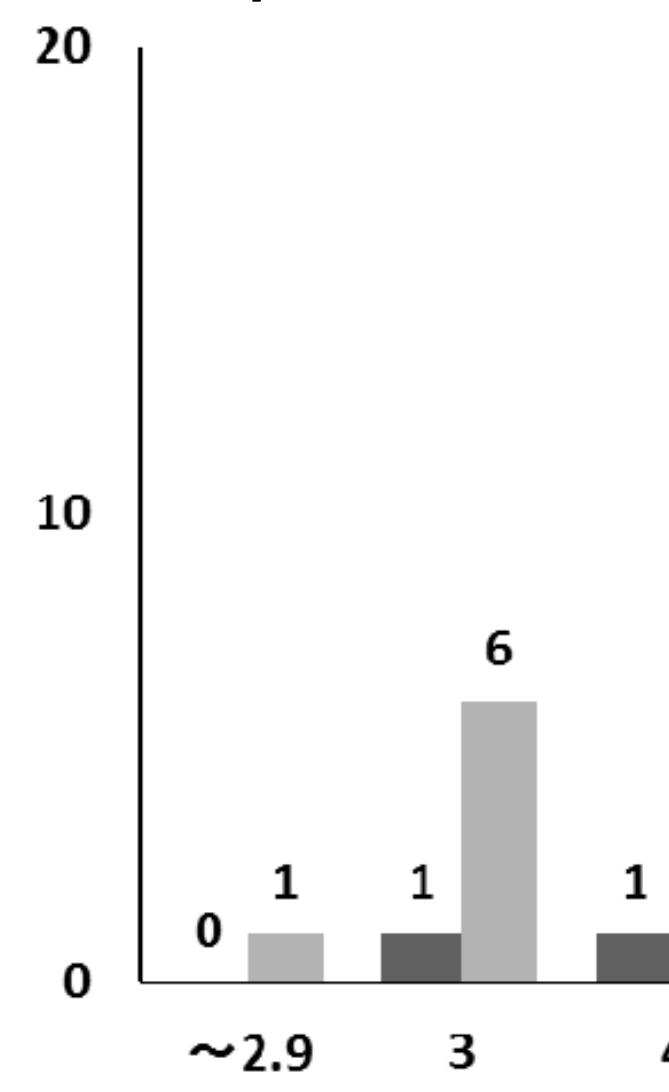

bone matching prostate/maker matching

11 


\section{Figure 6}

(b)

No. of Hospitals

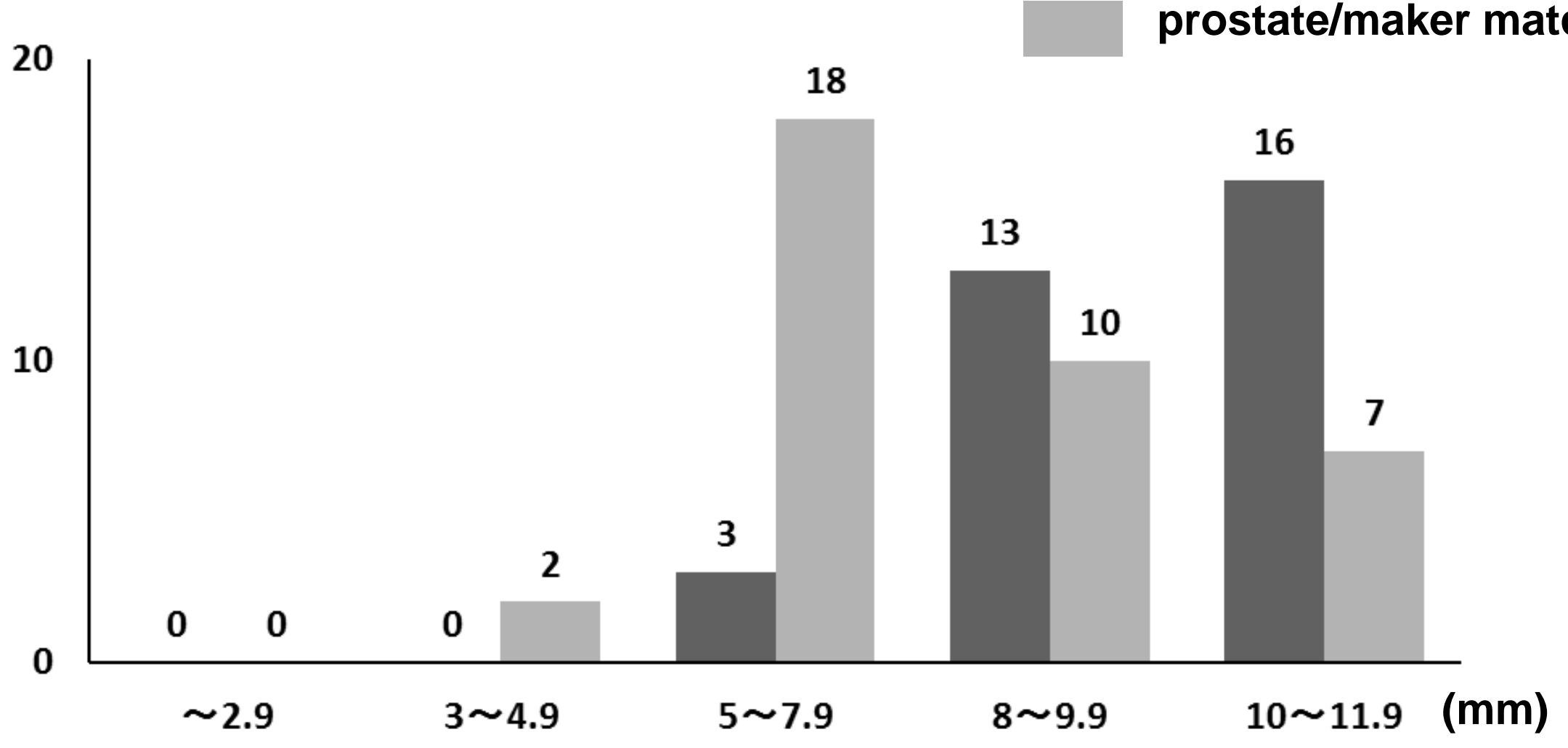

